\title{
Análisis comparativo de los aspectos económicos y sociales que caracterizan a los paises bolivarianos: retos $y$ expectativas ${ }^{1}$
}

\author{
Comparative analysis of the economic and social aspects that \\ characterize the bolivarian countries: challenges and expectations
}

\section{RESUMEN}

Antonio Caballero Tovio ${ }^{2}$

Este artículo tiene como propósito analizar y comparar los aspectos económicos y sociales de los países bolivarianos como son Bolivia, Colombia, Ecuador, Panamá, Perú y Venezuela, para identificar las similitudes y diferencias enmarcadas a lo largo de su historia; de igual forma determinar los retos a los cuales se enfrentan actualmente, y por otra parte conocer las perspectivas que se vislumbran en el mediano y largo plazo. Para tal propósito se utilizó como marco metodológico un estudio de tipo analítico-descriptivo, con un enfoque cuantitativo y cualitativo, utilizando

\footnotetext{
1 Este artículo se realizó debido a la inclinación del autor por esta clase de temas macroeconómicos, trabajados a través de los 29 años de experiencia en la materia, desarrollados y expuestos en artículos, clases, conferencias y conversatorios, en diferentes universidades; además por lo interesante que es comparar la tendencia de cinco naciones que tienen un origen común, con los ideales iniciales del libertador Simón Bolívar.

2 Economista de la Universidad de Cartagena, Especialista en Administración Financiera de la Universidad del Atlántico, Especialista en Gerencia Educativa Universidad de San Buenaventura, Especialista en Proyectos de Investigación y Formación Investigativa en el Servicio Nacional de Aprendizaje SENA, Consultor y Asesor Empresarial y Académico. Docente Investigador de la Corporación Universitaria Rafael Núñez. Autor de libro y artículos científicos.

Correo electrónico: antoniocaballero@curnvirtual.edu.co

Vicerrector Administrativo y Financiero de la Universidad de San Buenaventura, Decano, Director de programa, Director de especializaciones, Gerente de Fondo de Empleados, Docente Universitario.

(C) Los autores. Este artículo es publicado por Pensamiento Crítico de la Facultad de Ciencias Económicas, Universidad Nacional Mayor de San Marcos. Este es un artículo de acceso abierto, distribuido bajo los términos de la licencia Creative Commons Atribucion - No Comercia_Compartir Igual 4.0 Internacional. (http:// creativecommons.org/licenses/by-nc-sa/4.0/) que permite el uso no comercial, distribución y reproducción en cualquier medio, siempre que la obra original sea debidamente citada.
} 
y procesando estadísticas sobre el Producto Interno Bruto PIB, Ingreso Per cápita, Tasa de variación del PIB, Balanza Comercial, Inflación, Tasa de desempleo, y el Índice de Desarrollo Humano IDH .Este análisis permitió conocer con pensamiento crítico, los rasgos comunes y diferencias existentes entre estos países, lo que a su vez sentó las bases para una visión prospectiva en cuanto al desarrollo de las economías de estos países. Para tal propósito, primero se analizaron cada una de estas variables económicas, luego se generó la respectiva discusión, y por último las apreciaciones y conclusiones del caso.

Palabras clave: Aspectos económicos y sociales; Producción; Perspectiva global; desarrollo económico.

Clasificación JEL: Z13, E23, F01, F63.

\section{ABSTRACT}

The purpose of this article is to analyze and compare the economic and social aspects of Bolivarian countries such as Bolivia, Colombia, Ecuador, Panama, Peru and Venezuela, to identify the similarities and differences framed throughout their history; In the same way, determine the challenges they are currently facing, and on the other hand, know the expectations that can be glimpsed in the medium and long term. For this purpose, an analytical descriptive study was used as a methodological framework, with a quantitative and qualitative approach, using and processing statistics on Gross Domestic Product GDP, Per Capita Income, GDP variation rate, Commercial Balance, Inflation, Rate of unemployment, and the IDH Human Development Index. This analysis allowed to know with critical thinking, the common features and differences between these countries, which in turn laid the foundations for a prospective vision in terms of the development of the economies of these countries. For this purpose, each of these economic variables was analyzed first, then the respective discussion was generated, and finally the findings and conclusions of the case.

Keywords: Economic and social aspects; Production; Global perspective; economic development.

\section{Introducción}

Después de la primera crisis financiera del siglo XXI sucedida entre 2008 y 2009, a raíz del estallido de la burbuja inmobiliaria en Estados Unidos de América "EE.UU." y que se propagó por todo el mundo, se han observado cambios muy relevantes en el comportamiento de las economías a nivel mundial, caracterizados especialmente por una desaceleración de las economías desarrolladas, y un mayor crecimiento de las economías consideradas como emergentes. Al respecto, Caballero (2017) considera, 
que la crisis maltrató considerablemente a los países desarrollados, debido a que disminuyó notablemente la variación de su Producto Interno Bruto-PIB.

En el caso de los países bolivarianos es de suma importancia conocer con certeza como ha sido el comportamiento económico y social a lo largo de su historia y analizar la relación que existe entre dichas economías, de tal forma que se pueda establecer un horizonte claro sobre el futuro de estas naciones que de una u otra forma mantienen algunos elementos comunes como también diferencias en cuanto a la forma como han implementado sus estrategias económicas.

Los problemas que presentan estos países son muy complejos, a pesar de tener orígenes comunes, como el caso de Venezuela con un gobierno socialista que no convenció a la humanidad, arrastrando con la inflación más alta del mundo, o Colombia quien ha tratado de salir adelante con el peso de un conflicto armado, con grupos al margen de la ley, pero enmarcado en un proceso de paz financiado con altos impuestos a su población; en el caso de Perú no se ha visto claramente redistribuida la riqueza, de acuerdo como afirma Quispe (2017, p.1), “En el Perú, en el período 2009-2015, tanto la pobreza monetaria como la pobreza extrema monetaria a nivel nacional han disminuido, no obstante, los niveles de pobreza rural aún son muy altos, la pobreza es mayor en la sierra que en la selva y la costa".

Por otra parte, en lo referente al Índice de Desarrollo Humano - IDH, que expide las Naciones Unidas, éste indica que la calidad de vida de los bolivianos es regular, pues su índice de 0,674 se encuentra entre el rango de 0,50-0,80 establecido por el Programa de las Naciones Unidas para el Desarrollo - PNUD (2016), ocupando el nivel 118 en el mundo, a pesar que su PIB se incrementó un 4,3\% en 2016 Banco Mundial (2016); y si es Ecuador y Panamá con unas economías dolarizadas de acuerdo a sus características propias, se debaten en dos ámbitos similares, el primero en medio de una economía un poco más estabilizada, pero sin solucionar el problema de la pobreza y su desigualdad social, y el segundo con una economía moderna inmersa en medio de paraísos fiscales, con un buen crecimiento, pero dicha expansión no ha podido disminuir la 
pobreza y que de igual forma no logra mejorar la desigualdad social por el encarecimiento en el costo de vida de la población.

Pero lo anterior es apenas una anotación entre otras tantas, por lo cual se considera muy importante este documento por la manera como se desarrollará a continuación un análisis comparativo con pensamiento crítico y prospectivo basándose en la observación de las principales variables económicas y sociales de los países bolivarianos con el propósito de conocer sus características, retos y expectativas, y enmarcarlas en el contexto al cual pertenecen.

Se entiende por expectativas, a la esperanza de alcanzar un determinado propósito en el futuro; la conducta de ellos, dependerá de cuáles sean sus anhelos, de tal forma, que enfocadas en el ámbito económico y social, inciden directamente en la producción y en el consumo. Tal es el caso palpable que implican las demandas salariales de los trabajadores, y la disposición de los empresarios por conceder un determinado porcentaje de incremento salarial, todo dependerá de las expectativas que ambos tengan respecto comportamiento de la inflación para el próximo periodo.

Las Variables económicas y sociales que se utilizaron en este documento fueron el PIB, Tasa de Variación del PIB, Producto Interno Bruto Per cápita, IDH, Inflación, Tasa de desempleo, Balanza Comercial, Población, y Gasto en salud.

A continuación se muestran y analizan las relaciones de cada una de las variables antes mencionadas en estos países, con el propósito de identificar aspectos comunes y diferencias, contextualizadas en el ámbito macroeconómico.

\section{Análisis de las variable macroeconómicas en los países bolivarianos}

Se iniciará este análisis con el PIB, el cual se define como el total de bienes y servicios producidos en un país durante un período de tiempo determinado. Encierra la producción generada por nacionales residentes en el país y por extranjeros residentes en el país, y excluye la producción 
de nacionales residentes en el exterior Banco Mundial (2017). El PIB es la mejor medida del crecimiento económico de un país.

Se observa claramente que el PIB más alto entre los países bolivarianos los tiene Colombia y Perú lo que indica la mayor producción generada, siendo el PIB más bajo el de Bolivia. Lógicamente que esta variable es necesario relacionarla con el número de población, puesto que su relación es directamente proporcional; sin embargo, se puede utilizar para este caso, la tasa de variación anual del PIB, la cual muestra claramente que este indicador es mayor en Bolivia que en el caso de Colombia y Perú. En el caso de Venezuela la cual presenta un caso muy sui géneris, y sobre la cual se hará un análisis particular más adelante, presenta el tercer PIB entre estos países, pero con una tasa de crecimiento negativa. Por otra parte la tasa de crecimiento de Ecuador es baja; y como caso curioso Panamá con la menor población ha venido presentando el mayor crecimiento entre estos países (Ver Gráficos 1 y 3).

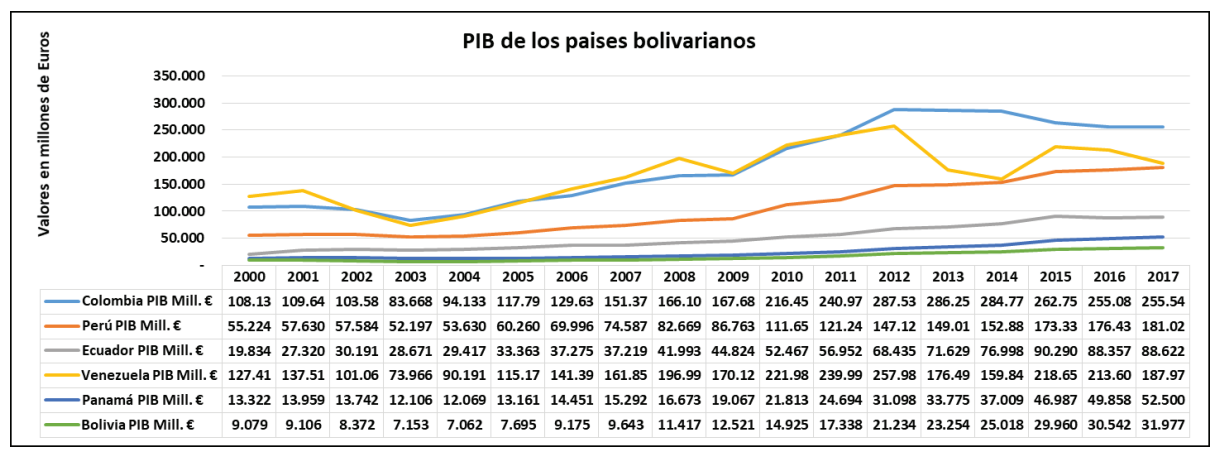

Gráfico 1. Producto Interno Bruto - PIB

Fuente: Datos procesados por el autor con base en información suministrada por Banco Central de Perú BCP, Banco Central de Ecuador BCE, Banco Mundial BM, Índex Mundi y Datos Macro.

En cuanto a la tendencia que estos países vienen presentando entre el año 2000 y el 2017, se observa que la mayoría de los países bolivarianos han venido manteniendo una producción estable con algunas pequeñas alteraciones a lo largo de estos años, a excepción de Venezuela, quien viene presentando una recesión económica caracterizada por crecimientos 
negativos a raíz de la crisis que esta nación viene atravesando desde el 2008 (Ver gráfico 3), pero que por supuesto tienen sus raíces desde hace aproximadamente 20 años.

El deterioro en la tasa de crecimiento del PIB de Venezuela ha generado una escasez con su secuela caracterizada por una hiperinflación ${ }^{3}$ la cual a su vez ha deteriorado el valor real del salario de sus habitantes

También es importante resaltar la baja tasa de crecimiento de Ecuador en $2015(0,20 \%)$ hasta tal punto que llegó a presentar un crecimiento negativo en 2016 a raíz de la baja en el precio de petróleo, a los altos impuestos, y en gran parte el terremoto del 16 de Abril de 2016.

Por otra parte es destacable el esfuerzo del Perú por mantener una buena evolución de su PIB al superar algunos inconveniente trascendentales desde el 1995 cuando el gobierno tuvo que enfrentar la crisis asiática, la salida de capitales y el fenómeno del niño, para luego al iniciar el siglo XXI luchar contra la inestabilidad política y el estancamiento de la inversión privada, y por último como si aún fuera poco el estallido de la crisis financiera mundial en 2009, con la caída en el precio de las materias primas; pero a pesar de todos estos inconvenientes hoy día Perú presenta un PIB entre los mejores de Latinoamérica, y su tasa de crecimiento aunque no es alta, su proyección a mediano y largo plazo es más clara que otros países, inclusive a nivel mundial.

Siguiendo en el análisis de las principales variables macroeconómicas de los países bolivarianos, es indispensable observar como el PIB per cápita corrobora la situación por la cual atraviesan estos países.

El PIB Per cápita, es aquel que muestra la cantidad promedio que le corresponde a cada habitante respecto a la producción de un país en un periodo determinado, generalmente de un año. Este indicador se ve influenciado y por lo tanto tiene relación directa con el nivel de la población de cada país, pero indudablemente muestra como podría ser la calidad de vida en un lapso de tiempo específico, claro está en combinación con el análisis del IDH.

3 La Hiperinflación consiste en un alza constante del nivel de precios, provocada generalmente por una disminución en el crecimiento de la producción y excesiva expansión del dinero en circulación generando un significativo desequilibrio entre la oferta y la demanda, deteriorando el poder de compra del dinero. 


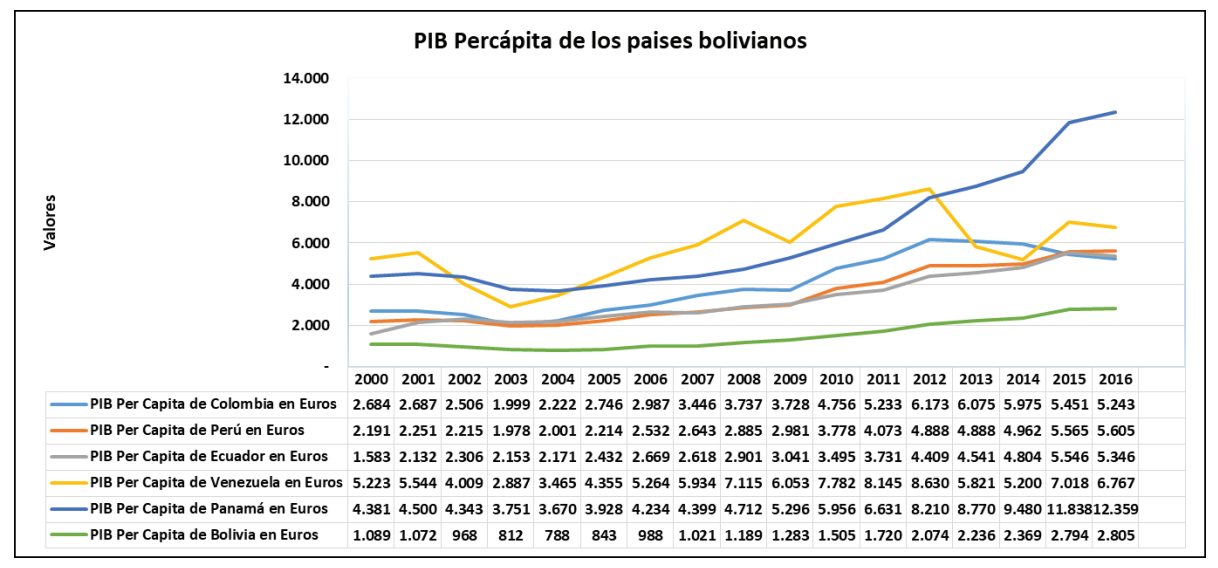

Gráfico 2. Ingreso Per Cápita

Fuente: Datos procesados por el autor con base en información suministrada por Banco Central de Perú BCP, Banco Central de Ecuador BCE, Banco Mundial BM, Índex Mundi y Datos Macro.

En el gráfico 3, se observa que el PIB per cápita más bajo a través de todos estos años es el de Bolivia, a pesar de tener una población relativamente baja en comparación con Colombia y Perú; los demás países bolivarianos han venido reflejando un incremento en su PIB per cápita, destacándose panamá el cual ha mostrado cifras bastante significativas como fueron de 11.838 y 12.359 euros durante el 2015 y 2016 respectivamente.

En el caso de Venezuela, ésta mostró en 2005 el PIB per cápita más alto de Latinoamérica, pero, luego, la economía empezó a manifestar una decaída vertiginosa con cifras tergiversadas, puesto que su PIB Per cápita aparentemente alto, no refleja una situación real, con unos resultados aparentes, que no deben impresionar a los espectadores, debido a que corresponden al resultado de una economía netamente extractiva, basada fundamentalmente en el petróleo y que sesgan la calidad de vida real de sus habitantes, porque en realidad su producción es decadente, hasta tal punto que hoy día la recesión económica y social es exagerada con una hiperinflación como la mayor del mundo.( Ver gráfico 4 , y el Ítem No.3 ) 


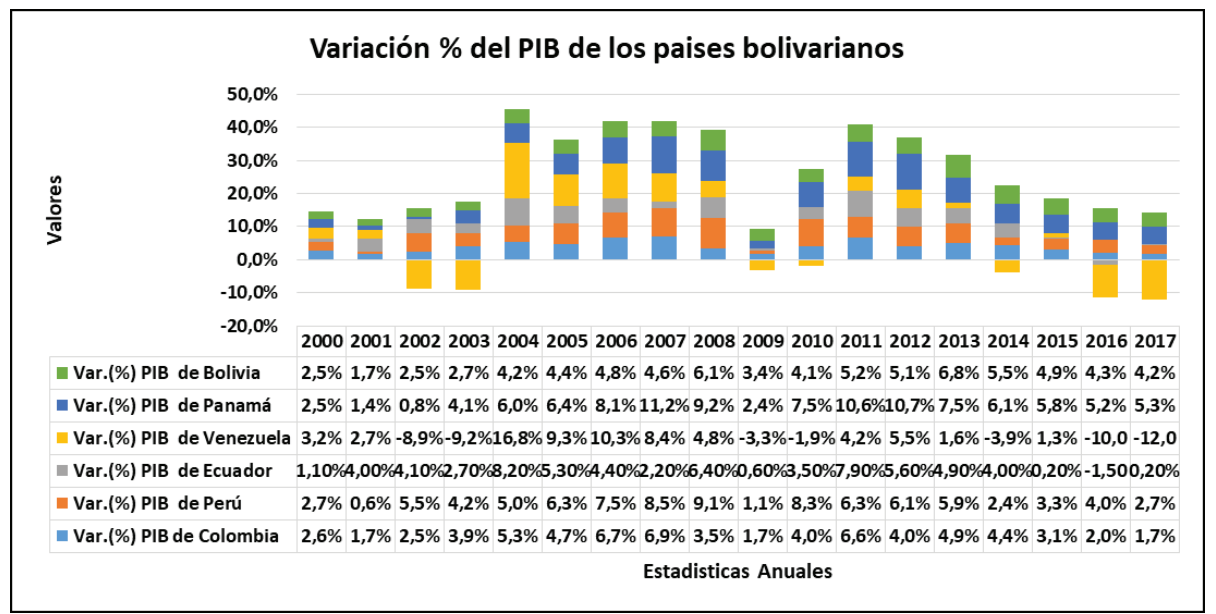

Gráfico 3. Tasa de Variación del PIB

Fuente: Datos procesados por el autor con base en información suministrada por Banco Central de Perú BCP, Banco Central de Ecuador BCE, Banco Mundial BM, Índex Mundi y Datos Macro.

En el gráfico 4, se puede apreciar los niveles de inflación de las economías bolivarianas, destacándose un alto grado de polarización: en el caso de Venezuela con una desproporcionada inflación, y el otro caso corresponde a Ecuador y Panamá, los cuales presentan la inflación más baja entre estos países, lo cual está muy relacionado con la dolarización de sus economías y ha permitido su estabilización; desde hace 18 años Ecuador implementó el dólar como moneda oficial, cuando en el año 2000 presentaba una inflación del orden del 96\%, y de ahí en adelante ha venido mostrando una tendencia totalmente a la baja, hasta tal punto que en 2017 su inflación fue del orden del 0,7 \% ; de igual manera Panamá ha venido reflejando un comportamiento bastante favorable evidenciado por bajas tasas de inflación anual. En resumidas cuentas, tal como dicen Herrera \& Caballero (2002), la dolarización de la economía es aquel proceso mediante el cual, la moneda extranjera sustituye concretamente a la original de un país. Por otro lado, Muñoz (2002) reflexiona al respecto y opina que la dolarización representa el acogimiento por parte de una nación, ya sea de manera individual o pactada, de la moneda de un país extranjero. Y en este mismo orden de ideas, Schuler (1999), considera que para ciertas naciones, la moneda autóctona es un elemento imprescindible 
para la identidad nacional, al prescindir la moneda propia y reemplazarla por el dólar se derrocha parte de esa identidad.

Con respecto a lo antes mencionado, todos los autores logran coincidir en que la dolarización se ha efectuado tradicionalmente para reparar los desequilibrios macroeconómicos que muestran las economías, tales como, los efectos inflacionarios, un perturbado movimiento en los tipos de cambio, para desequilibrios fiscales y en cuenta corriente, y de desempleo, entre otros.

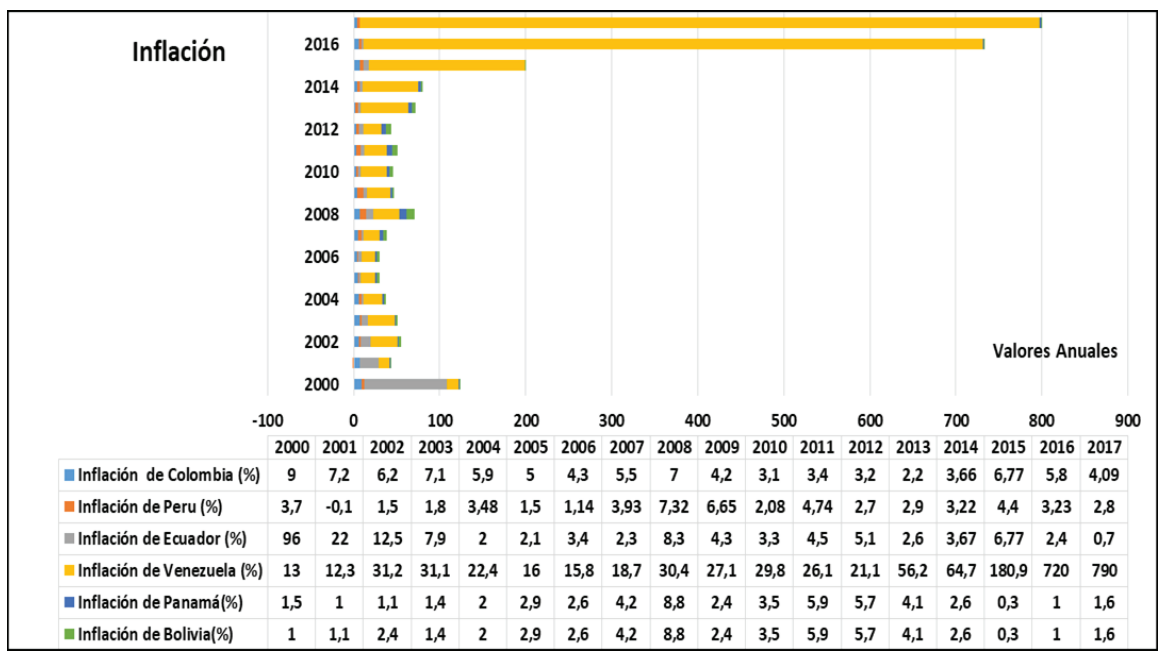

Gráfico4. Inflación

Fuente: Datos procesados por el autor con base en información suministrada por Banco Central de Perú BCP, Banco Central de Ecuador BCE, Banco Mundial BM, Índex Mundi y Datos Macro.

Le siguen en el mismo orden Bolivia, Perú y por último Colombia con una tasa de 4,09 en 2017; en el caso de Colombia la inflación se está manteniendo relativamente alta, debido a que esta economía, con la firma del Tratado de Libre Comercio - TLC con los Estados Unidos E.E.U.U. ha venido afectándose por la revalorización del Dólar, puesto que al hacer un balance de este TLC, se detectó que si bien es cierto que han aumentado la diversificación de productos exportados por Colombia a los E.E.U.U, también es muy cierto que ha sido mucho mayor el incremento de sus importaciones, que el de las exportaciones, y el déficit en la Balanza 
Comercial de Colombia con estado Unidos se inició exactamente en el año que empezó a operar este TLC, tal como lo comenta Perdomo ( 2016), lo cual ha generado un elevado costo para el consumidor, y un deterioro constante de los ingresos del sector familia, por la revaluación del dólar.

Por otra parte, el comportamiento de la Balanza Comercial de los países bolivarianos mostrada en la gráfico 5, refleja la diferencia entre las exportaciones y las importaciones de estos países durante un periodo determinado, generalmente de un año, y a través de ella se puede conocer el superávit o déficit en las transacciones internacionales.

En el gráfico 5, se aprecia que en el presente caso, este indicador es bastante complejo, por el comportamiento irregular que muestra la tendencia de los países bolivarianos entre el 2004 a 2016; sin embargo se puede afirmar que Perú siempre ha mantenido en esos periodos una balanza comercial superavitaria, a excepción de los años 2013, 2014 y 2015, debido a la caída de sus exportaciones mineras como el cobre y el zin, causado por el estancamiento de la economía china y europea, además de la disminución de sus exportaciones tradicionales, sin embargo en estos momentos ya Perú ha superado esos continuos déficit, y en 2016 presentó un superávit en las transacciones internacionales; y actualmente están haciendo arduos esfuerzos por diversificar sus exportaciones e incursionar en nuevos mercados trabajando, en forma conjunta entre el sector oficial y privado.

En este mismo orden de ideas, la balanza comercial de Ecuador se vio muy lesionada con la caída en el precio del petróleo a partir de 2008, pues como es sabido, la demanda a nivel global se redujo a raíz de que E.E.U.U encontró un pozo con formación de roca sedimentaria muy rica en esquisto y shale oúl, lo que ha hecho que esta nación que era uno de los mayores importadores de petróleo, a partir de 2015 empezó a producir 9,3 millones de barriles por día, lo que supondría un incremento diario de 700.000 barriles respecto a la media de 2014, tal como se comenta en un informe en El Economista (2014). Ya en 2016, Ecuador muestra un superávit en su balanza comercial debido al incremento de la Inversión extranjera IED, a la cual el gobierno le garantizó seguridad jurídica, además de la reducción de las importaciones con salvaguardia a estas, y 
acuerdo de multipartes, medidas consideradas como muy acertadas por el Centro Estratégico Latinoamericano de Geopolítica - CELAG (2016).

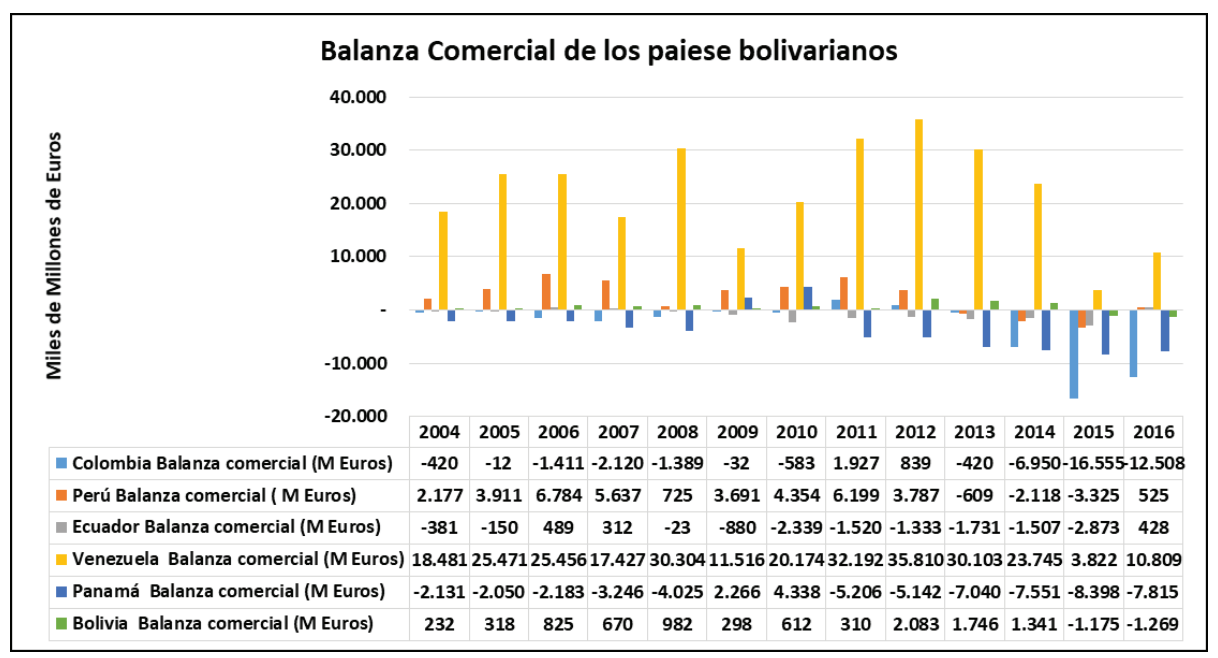

Gráfico 5. Balanza Comercial

Fuente: Datos procesados por el autor con base en información suministrada por Banco Central de Perú BCP, Banco Central de Ecuador BCE, Banco Mundial BM, Índex Mundi y Datos Macro.

En el caso de Bolivia, se nota que éste país ha venido aumentando cada vez más su déficit en la balanza comercial, debido a la afectación que tuvo el precio del gas natural que Bolivia exporta a Argentina y a Brasil; además las medidas proteccionistas del presidente de los E.E.U.U Donald Trump, la desaceleración de la economía China, y la salida de Reino Unido del mercado común europeo han entorpecido la fluidez del comercio de sus productos en estos mercados, de acuerdo con lo anotado por Olmo (2017).

En esta secuencia analítica, Colombia presenta el mayor déficit en su balanza comercial entre los países bolivarianos, seguida de Panamá. La situación de Colombia es muy clara si se tiene en cuenta que con la firma del TLC con los E.E.U.U. este país se ha visto afectado por la reducción de precio del petróleo y la revaluación del dólar, puesto que sus importaciones han aumentado más que las exportaciones; de tal manera que las compras 
en dólares han generado una inflación importada, la cual se trasfiere al consumidor final.

En cuanto a Panamá, el déficit se debe en gran parte por las medidas proteccionistas de Colombia para las prendas de vestir y para el calzado en general, y además, a la carencia de pagos de los importadores venezolanos, de acuerdo con lo expresado por Varela (2016); a esto se le suma la baja en los precios de productos tradicionales como el banano y azúcar. Por otra parte, De Ycaza (2016), considera que la IED compensa el déficit comercial en este país.

Es curioso como Venezuela mantienen unos indicadores por encima de la mayoría de los países bolivarianos, cuando en realidad todo obedece a la metodología utilizada para esos cálculos, puesto que es muy lógico que un país que mantiene la inflación más alta del mundo es porque no está produciendo los bienes y servicios que su población está demandando, lo cual hace que los precios se disparen, y se presente la hiperinflación actual en este país; por otra parte, y en la misma secuencia, si este país no está produciendo los bienes y servicios necesarios para satisfacer las necesidades básicas de sus habitantes, mucho menos puede generar excedentes para mantener un nivel de exportaciones alto.

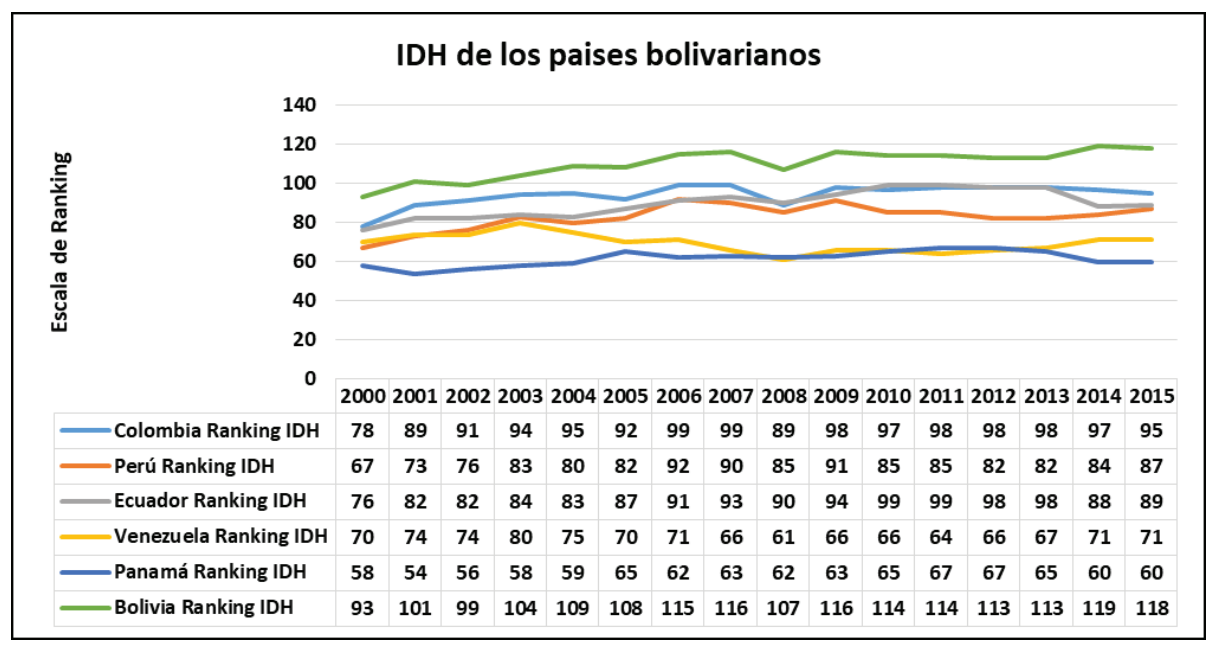

Gráfico 6. Índice de Desarrollo Humano IDH

Fuente: Datos procesados por el autor con base en información suministrada por Banco Central de Perú BCP, Banco Central de Ecuador BCE, Banco Mundial BM, Índex Mundi y Datos Macro. 
De acuerdo con el anterior análisis, apoyado en las estadísticas de los gráficos expuestas en este artículo, se puede afirmar que Venezuela mantiene una balanza comercial aceptable a raíz de la extracción y exportación de petróleo, pero dichos petrodólares no están dirigiéndose a elevar el nivel de vida de su población; pues es muy sabido que la falla principal del sistema económico de Venezuela es la poca producción. Además el desempleo también es alto ( Ver gráfico7), debido a que no se está utilizando su capacidad productiva en forma adecuada, lo cual unido a la alta tasa de inflación (Gráfico 4), genera un elevado costo en el nivel de vida de tal forma que las variables como educación, salud y esperanza de vida no están acordes con la realidad que el país está atravesando, considerándose como se mencionó anteriormente metodologías inadecuadas en los cálculos de los indicadores socioeconómicos, como por ejemplo el IDH.

El IDH es un indicador que fue ideado por el Programa de las Naciones Unidas para el Desarrollo PNUD con el fin de determinar el nivel de desarrollo que tienen los países del mundo para lo cual se analizan las dimensiones de salud, educación, y esperanza de vida digna. En el gráfico 6, se puede apreciar claramente que todos los países bolivarianos presentan un IDH regular, siendo el menos favorecido el de Bolivia. Sin embargo estas naciones mantienen una alta tasa de pobreza, tal como se aprecia en el cuadro 3.

Continuando las apreciaciones relacionadas con las variables socioeconómicas de los países bolivarianos, es bueno aclarar que por lo complejo del caso, se requiere utilizar cada una de las variables en forma irregular, es decir sin un orden especifico, sino de acuerdo con las circunstancias que se presentan para cada país y su relación con el total.

En cuanto a la tasa de desempleo, se puede notar que Venezuela mantiene una tasa del orden del 26,4\% (ver gráfico 7), lo cual es muy entendible de acuerdo con lo analizado en párrafos anteriores, y que tiene que ver directamente con el real nivel de vida, muy bajo, que actualmente presenta este país, lo cual ha generado el éxodo de venezolanos hacia otros países, especialmente a Colombia. 
En este mismo orden de ideas, y de acuerdo con lo expuesto por Hernández (2015), es un axioma económico, que si el Gobierno de Venezuela hubiese generado garantías a la inversión privada, y si, con la bonanza originada por los petrodólares se hubieran creado empresas productoras de bienes y servicios para cubrir la demanda de su población, hoy día, este país, no estuviera arrastrando con la mayor inflación del mundo y el gran éxodo de sus habitantes.

Como caso curioso Colombia sigue a Venezuela en ese mismo orden con una tasa de las más altas entre estos países, que alcanzó en 2017 a 9,4\%, mientras que los otros países bolivarianos oscilan entre un 4\% y un $6,7 \%$.

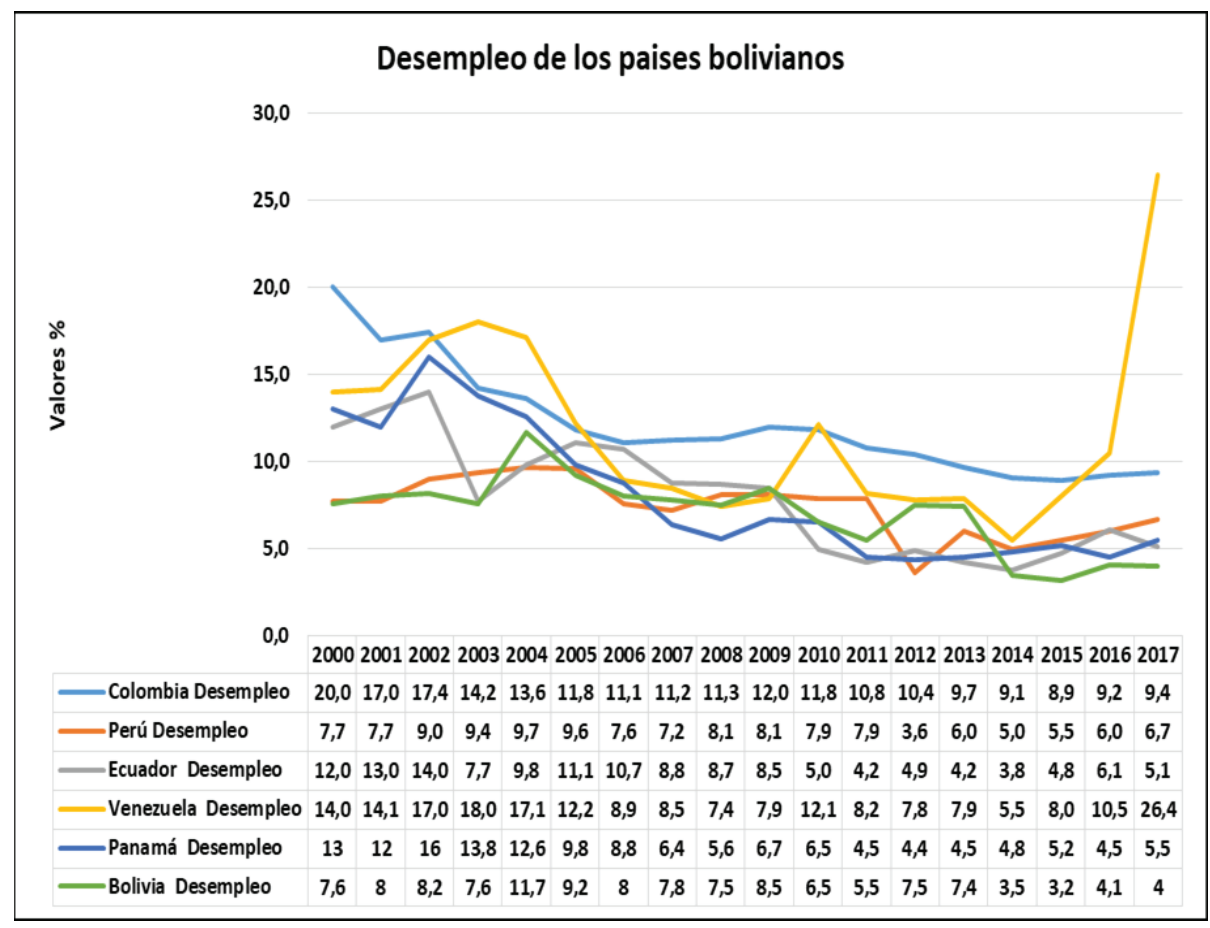

Gráfico 7. Tasa de Desempleo

Fuente: Datos procesados por el autor con base en información suministrada por Banco Central de Perú BCP, Banco Central de Ecuador BCE, Banco Mundial BM, Índex Mundi y Datos Macro. 
En cuanto a la tasa de desempleo en los países bolivariano es importante considerar que la economía informal ha aumentado considerablemente, y el criterio que se utiliza para aplicar la metodología de cálculo es bastante imprecisa y ambigua, sin embargo muchos de estos países afirman que la metodología utilizada está acorde con las normas establecidas por la Organización Mundial del Trabajo - OIT; en el caso de Colombia, como afirma Perfetti (2014), un trabajador que vende dulce durante más de una hora a la semana, en un transporte, o en la calle, y recibe cualquier remuneración, se considera empleado; en Bolivia, aunque presenta la tasa de desempleo más baja entre este grupo de países, el empleo de mala calidad o efímero se amplió por el incremento del sector informal, que llega a 70\% de los ocupados, Rojas (2014); y generalmente esto es un fenómeno que ha venido sucediendo en este grupo de países, y peor aún, que a raíz del alto nivel de pobreza existente, se ha acrecentado en los últimos 20 años.

Cuadro 1. Población de los países bolivarianos 2015

\begin{tabular}{lcc}
\multicolumn{1}{c}{ País } & Población & Fuente \\
\hline Colombia & 48.203 .405 & DANE \\
Perú & 31.151 .643 & INEI \\
Ecuador & 14.473 .169 & INEC \\
Venezuela & 30.825 .782 & INE \\
Panamá & 4.058 .372 & INEC \\
Bolivia & 10.825 .000 & INE \\
\hline
\end{tabular}

Fuente: elaboración propia basado en los datos que proporcionan el Departamento Nacional de Estadísticas DANE,( Colombia) Instituto Nacional de Estadísticas e Informática INEI (Perú), Instituto Nacional de Estadísticas y Censo INEC (Ecuador), Instituto nacional de Estadística INE ( Venezuela Panamá y Bolivia). Se escogió el 2015 debido a que en ese año había censo común.

Algo que corrobora el análisis del IDH en estos países, es el gasto que estos países dedican a la salud de sus habitantes relacionándolo como porcentaje de su PIB; en el cuadro 2, se aprecia que aunque este porcentaje (Indicador) ha venido mejorando en estos países paulatinamente, a excepción de Venezuela, aún sigue siendo un problema por solucionar el nivel de pobreza en estas naciones, (Cuadro 3). 
Cuadro 2. Gasto en salud como porcentaje del PIB (1999-2014)

\begin{tabular}{lcccccc} 
Fecha & $\begin{array}{c}\text { Colombia G. } \\
\text { Salud \%PIB }\end{array}$ & $\begin{array}{c}\text { Perú G. } \\
\text { Salud \%PIB }\end{array}$ & $\begin{array}{c}\text { Ecuador G. } \\
\text { Salud \%PIB }\end{array}$ & $\begin{array}{c}\text { Venezuela } \\
\text { G. Salud } \\
\text { \%PIB }\end{array}$ & $\begin{array}{c}\text { Panamá G. } \\
\text { Salud \%PIB }\end{array}$ & $\begin{array}{c}\text { Bolivia G. } \\
\text { Salud \%PIB }\end{array}$ \\
\hline 1999 & $5.37 \%$ & $2.83 \%$ & $1.38 \%$ & $1.83 \%$ & $4.54 \%$ & $3.60 \%$ \\
2000 & $4.68 \%$ & $2.72 \%$ & $0.90 \%$ & $2.16 \%$ & $5.28 \%$ & $3.25 \%$ \\
2001 & $4.69 \%$ & $2.83 \%$ & $1.23 \%$ & $2.34 \%$ & $5.18 \%$ & $3.74 \%$ \\
2002 & $4.55 \%$ & $2.83 \%$ & $1.43 \%$ & $2.15 \%$ & $5.54 \%$ & $4.10 \%$ \\
2003 & $4.90 \%$ & $2.69 \%$ & $1.43 \%$ & $2.08 \%$ & $5.05 \%$ & $3.50 \%$ \\
2004 & $4.08 \%$ & $2.54 \%$ & $1.43 \%$ & $2.16 \%$ & $5.69 \%$ & $3.49 \%$ \\
2005 & $4.32 \%$ & $2.69 \%$ & $1.31 \%$ & $2.15 \%$ & $5.21 \%$ & $3.86 \%$ \\
2006 & $4.54 \%$ & $2.39 \%$ & $1.42 \%$ & $2.22 \%$ & $4.72 \%$ & $3.52 \%$ \\
2008 & $4.54 \%$ & $2.41 \%$ & $1.51 \%$ & $2.46 \%$ & $4.05 \%$ & $3.41 \%$ \\
2009 & $4.70 \%$ & $2.45 \%$ & $1.63 \%$ & $1.23 \%$ & $4.67 \%$ & $3.41 \%$ \\
2010 & $5.15 \%$ & $2.98 \%$ & $2.19 \%$ & $2.50 \%$ & $5.63 \%$ & $3.95 \%$ \\
\hline 2013 & $4.98 \%$ & $2.79 \%$ & $2.46 \%$ & $2.09 \%$ & $5.65 \%$ & $3.66 \%$ \\
\hline & $5.02 \%$ & $2.62 \%$ & $2.60 \%$ & $2.34 \%$ & $5.09 \%$ & $3.75 \%$ \\
\hline
\end{tabular}

Fuente: Elaboración propia basado en los datos que proporcionan el DANE, INEI, INEC, INE; Se escogió el 2015 debido a que en ese año había censo común.

Cuadro 3. Tasa bajo el nivel de pobreza

País

Colombia

Ecuador

Panamá

Perú

Bolivia

Venezuela

\section{(\%) Años entre 2014 y 2015}

28

26

23

23

39

20

Fuente: elaboración propia basado en información suministrada por Índex Mundi 


\section{Apreciaciones y Conclusiones}

En este ítem, el autor presenta una serie de apreciaciones referentes a cada uno de estos países analizados a lo largo del documento, utilizando un enfoque de crítica constructiva, y a la vez de una manera concluyente, por las características propias del tema.

\subsection{Colombia}

En relación a la economía de Colombia, el pulso de ésta ha bajado, debido a que por un lado, la débil demanda interna contribuyó a disminuir la inflación por debajo del límite superior del rango estipulado por el Banco de la República de Colombia en 2017, pero a su vez dicha disminución bajó el crecimiento económico por la reducción del nivel en su actividad económica. Esta desaceleración, junto con un replanteamiento de las expectativas, permitió al Banco de la República bajar la tasa de referencia en veinticinco puntos básicos a 5,25\% en septiembre de 2017, de acuerdo con información del DANE (2017).

No obstante, entre los países bolivarianos, la situación de Colombia es muy particular puesto que entre estos países, se puede afirmar, que es el que ha llevado la peor parte de los desastrosos efectos originados por los grupos armados, tales como la guerrilla, paramilitares y narcotraficantes, lo cual ha generado desde siempre, un freno al desarrollo del sector agroindustrial.

Así las cosas, y contrariamente a lo que se esperaba, que con el segundo mandato de Juan Manuel Santos en 2014, y después de cuatro años de negociación entre el gobierno de Colombia y las Fuerzas Armadas Revolucionarias de Colombia-Ejército del Pueblo (FARC-EP), que fue firmado en noviembre de 2017 por el presidente Santos y el dirigente de las FARC, Rodrigo Londoño 'Timochenko', hoy día, se vislumbra una incertidumbre en el futuro político y económico de esta nación, tal como lo comenta Gómez (2017)

Por otra parte, falta el acuerdo de paz con el Ejército de Liberación Nacional - ELN quienes siguen vulnerando la seguridad e integridad de sus habitantes; además hay que tener en cuenta que existen otros grupos al margen de la ley, como son las disidencias de la FARC, narcotraficantes y paramilitares. 
Lo anterior se corrobora con la tesis de Castro (2014), quien considera que el manejo adecuado de conflictos garantizará la convivencia de las futuras generaciones, e indudablemente, es una variable que incidirá en el nivel de desarrollo económico y social del país; recapitulando sobre lo anteriormente descrito, y respecto a la tesis de Castro, considero que resulta contraproducente y contradictorio, el hecho, que para cubrir el gasto que genera el pos conflicto, el gobierno ha elevado la carga fiscal a sus ciudadanos, lo cual ha ocasionado una disminución del poder de compra de las familias, con la consiguiente reducción de la demanda, y como es sabido una baja en la tasa de crecimiento del PIB. Situación ésta que por experiencia, de acuerdo con lo esbozado por Sarmiento (2017), ha generado recesiones en otros países como por ejemplo en E.E.U.U en 1929, cuando el presidente Hoover tuvo la necesidad de disminuir la tasa de impuesto con el fin de reactivar la economía

En tal sentido, el ajuste económico ante el importante choque petrolero continuó durante el 2016 y el crecimiento económico se desaceleró. Sin embargo, la economía colombiana ha sido resistente frente a este trauma. La situación económica fue más endeble, especialmente para las industrias extractivas, a raíz de una mayor incertidumbre, y a una pausada realización en los proyectos financiados con capitales públicos, lo cual produjo una mengua en la inversión.

Como en la mayoría de las veces, el sector servicios se mantuvo como el que más ayudó al crecimiento, debido a la actividad de los servicios financieros, comerciales y de la construcción, lo cual compensó en parte la caída del sector extractivo. Por otro lado, el sector agrícola se perjudicó significativamente por el fenómeno de El Niño.

En ese orden de ideas, el crecimiento ha sido más lento de lo esperado en 2017, con efectos de desconfianza que pesan sobre el consumo privado y la inversión, al igual que debilidad de la demanda externa, y cuestiones de competitividad que deterioran el normal desenvolvimiento de las exportaciones.

Como expectativa para el periodo entre 2017 a 2019, se espera un repunte en el crecimiento económico, promovido por la redención de las exportaciones no petroleras y los precios del petróleo, al igual que el programa de infraestructura de cuarta generación 4G. 


\subsection{Perú}

En lo relacionado con la República de Perú, en el transcurso de la última década, ésta ha sido una de las economías de más rápido desarrollo en la región, con una tasa de aumento promedio de 5.89 \% en un contexto de baja inflación. El entorno externo favorable, políticas macroeconómicas juiciosas y reformas estructurales en distintas áreas generaron un escenario de alto desarrollo y baja inflación, según lo muestran los datos del Banco Mundial - BM (2018).

Por otro lado, el efectivo aumento en el empleo y en los ingresos disminuyó grandemente las tasas de pobreza, por ejemplo, la pobreza moderada (US\$4,2 por día con un PPA del 2005) cayó de $45.7 \%$ en el 2005 a $19.4 \%$ en el 2015, lo cual corresponde a decir que 6.4 millones de personas salieron de la pobreza durante ese lapso de tiempo. La pobreza extrema (US\$2.4 por día con un PPA del 2005) disminuyó de 27.8 \% a $9,2 \%$ en ese tiempo, ya en 2016 , el porcentaje de la población peruana en escenario de pobreza extrema pasó de 4.1\% (cifra de 2015) a 3.8\%, de acuerdo con información de INEI (2017).

Con respecto a lo antes planteado, se puede inferir que Perú es una de las economías que en Latinoamérica muestra una tendencia hacia el crecimiento económico y social, con muchas oportunidades en su mercado exterior vislumbrándose una mejor calidad de vida para sus habitantes.

En ese mismo orden, el incremento del PIB siguió acelerándose en el 2016, amparado por mayores cantidades de exportación minera gracias a que una serie de proyectos mineros de gran envergadura ingresaron a su etapa de producción y lograron su volumen total.

De igual forma se aprecia que la economía prosperó por encima de su potencial a 3.92\% en el 2016, a causa del pico estacional de la manufactura minera, pero en 2017 volvió a disminuir su tasa de crecimiento, debido al estacionamiento en el sector minero, y a una débil inversión privada, la cual se vio afectada por las condiciones globales desfavorables y la incertidumbre relacionada con los casos de corrupción de proyectos firmados en años pasados. 
De acuerdo con lo anterior es importante considerar que las perspectivas del crecimiento de la economía peruana son sensibles a los impactos externos en relación a los precios del productor, la desaceleración del crecimiento de China, la volatilidad de los mercados de capital, y la velocidad del ajuste de la política monetaria en los Estados Unidos.

También es importante tener en cuenta, que esta economía está expuesta a los riesgos naturales, como son los fenómenos climáticos periódicos, como El Niño. Para compensar estas externalidades negativas y garantizar el crecimiento, se requiere de reformas estructurales y fiscales que faciliten la productividad, disminuyan la informalidad, y optimicen la eficiencia de los servicios públicos.

\subsection{Ecuador}

En lo referente a la República de Ecuador, se aprecia en el gráfico 3, que en el periodo de 2006 y 2014, experimentó un crecimiento promedio del PIB del orden del 4,34\%inducido por los altos precios del petróleo y de significativos flujos de financiamiento externo al sector público, lo que permitió un mayor gasto público, envolviendo la expansión del gasto social e inversiones representativas en los sectores de energía y transporte.

Durante ese mismo lapso de tiempo, la pobreza se redujo del 37,58\% al 22,46\% y el coeficiente de desigualdad se disminuyó de 0,52 a 0,47 originado por el hecho de que los ingresos de los estratos más necesitados de la población ascendieron más rápido que el ingreso promedio, pero estos resultados están en peligro debido a la baja de los precios del petróleo y la revaluación del dólar.

Como si fuera poco, todo el esfuerzo se ha visto descompensado por un terremoto que afectó la zona costera noroccidental del país en abril de 2016, incrementando el desempleo, y la pobreza; tal como se comenta en la revista Dinero (2016, p 1):" Antes del terremoto, el FMI había calculado una caída en la economía ecuatoriana cercana a 4,5\%, por efectos de los menores precios del petróleo y la pérdida de competitividad derivada de la dolarización", lo cual se corrobora en una edición del diario Gestión de Perú, en el cual se informa que dejaron de recibir US $\$ 7.000$ millones por cuenta de la caída en los precios internacionales del crudo. 
Así las cosas, en este contexto en que la inversión pública no podrá seguir siendo motor del desarrollo, es preciso un incremento sistemático en el clima de inversiones que induzca una mayor participación privada y acelere la movilidad del capital y del trabajo en actividades productivas.

Por lo tanto, es indispensable un sector privado más robusto y flexible que ayudaría a diversificar la economía ecuatoriana, acrecentar su productividad y crear empleos de calidad con el fin de suscitar el desarrollo y continuar con la contracción de la pobreza.

\subsection{Bolivia}

En lo que respecta a la economía de la República de Bolivia, se aprecia en el gráfico 3, que entre el 2004 y 2014, la economía de Bolivia prosperó a una tasa anual promedio del $4,92 \%$ inducida por los elevados precios de las materias primas y una estrategia macroeconómica sensata, lo que hizo que la pobreza se disminuyera de un 59,3\% al 39,2\%, durante los años de 2005 y 2014

Pero a consecuencia de un contexto internacional un poco adverso, el crecimiento del PIB se redujo de un pico de 6,8\% en 2013 a cerca de 4,3\% en 2016(Ver gráfico3), y la disminución de la pobreza y desigualdad ha perdido propulsión.

Con el propósito de mantener un desarrollo alto, continuar disminuyendo la pobreza y optimizar el acceso a servicios básicos, fue aceptado por el Gobierno de Bolivia, a principios de 2016, el Plan Nacional de Desarrollo Económico y Social (PDES) 2016-2020. CEPAL (2016), el cual incluye ambiciosos programas de inversiones públicas, financiadas por los ahorros, atesorados en la bonanza, créditos del Banco Central de Bolivia BCB y endeudamiento externo.

Estas inversiones vislumbran infraestructura, búsqueda de hidrocarburos, fabricación de gas natural y construcción de hidroeléctricas, al igual que contempla un mayor dinamismo del sector privado y de la inversión extranjera directa.

En este sentido, y con miras a robustecer su posición como exportador de gas natural a la región, Bolivia demanda unificar esfuerzos con el sector privado para acrecentar las reservas que tiene. En forma simultánea es 
inminente atraer inversiones en sectores que tradicionalmente han sido menos atractivos para los inversionistas privados, incluyendo minería, agricultura y manufactura.

\subsection{Panamá}

En lo referente a Panamá, de acuerdo con la Revista Forbes (2017), se espera que el sector privado apoye significativamente en áreas claves como transporte y logística, minería, servicios financieros, y turismo; de igual manera se aprecia que se han conseguido progresos importantes en la reducción de la pobreza en los últimos años, puesto que si se utiliza la raya de pobreza internacional de US 4,1 al día, reflejaría una disminución de la pobreza general de $21,3 \%$ a $16,8 \%$, en el periodo comprendido entre los años de 2011 a 2015. Lo que se ayuda con el hecho de que la economía ha generado 279 mil nuevos empleos.

En este mismo contexto, algo muy importante que se debe resaltar es que la economía Panameña, ha sido una de las economías de más rápido desarrollo en los últimos diez años, con un incremento medio anual del orden del 7\%, entre los años 2001 al 2013, (Ver gráfico 3). Esta economía creció un $6.1 \%$ en 2014, bajando levemente a un 5.82\%, 5,2\% y 5.3\% en 2015, 2016, y 2017 respectivamente, y en estos momentos representa la tasa de crecimiento del PIB más alta en Latinoamérica.

Es de esperar que este crecimiento se mantenga con el proyecto de construcción de la segunda línea del Metro y el comercio adicional originado por el ensanchamiento del Canal; se espera además, que las inversiones públicas también se conserven altas.

Sin embargo aún falta mucho por mejorar, puesto que la pobreza predomina en zonas rurales, generalmente habitadas por grupos indígenas; y, si no se afrontan algunas áreas prioritarias estructurales, podrían entorpecer el crecimiento en los próximos años; estas contemplan: infraestructura, educación y destrezas, así como la eficiencia de las instituciones públicas.

Como prueba al esfuerzo que se ha realizado por el gobierno están los indicadores del PIB, tasa de crecimiento, IDH, PIB per cápita, entre otros, como se observa en los gráficos expuestos a lo largo de este artículo. 
El Plan de Desarrollo Estratégico 2015-2019 del Gobierno de Panamá se basa en dos pilares de inclusión y competitividad e incluye cinco temas CEPAL (2015):

- Impulsar la productividad y diversificación del crecimiento.

- Mejorar la calidad de vida

- Fortalecer el capital humano

- Desarrollar la infraestructura

- Apoyar la sostenibilidad del medio ambiente, incluida la gestión.

\subsection{Venezuela}

De acuerdo con información del Banco Mundial (2017), se sabe que a principios de la década pasada, hasta fines del año 2014, la economía de la República de Venezuela se vio beneficiada por los precios del petróleo, históricamente altos, lo cual le permitió aumentar el gasto público en proyectos trascendentales.

A qué se debe la decadencia de la hermana República? De acuerdo con Giuman (2017), esto se originó, a que durante el mandato de Chávez, se fundaron diversas empresas oficiales, y nacionalizó un buen número de empresas privadas en sectores como hidrocarburos, minería, metalurgia, cemento, banca y telecomunicaciones, llevando a cabo, además, una amplia gama de programas sociales, con el propósito de suministrar servicios básicos y cambios de recursos a favor de sectores de la población marginada.

En forma simultánea, el desarrollo económico y la ejecución de políticas redistributivas facilitaron la disminución de la pobreza, en todas sus dimensiones, al igual que la desigualdad social, pero adversamente, la caída del precio internacional del petróleo, unido a las políticas desacertadas a nivel tanto macro como microeconómico, descompensaron de manera sustancial el desarrollo económico y social del país.

En este mismo orden de ideas, se puede afirmar que se considera una política bastante desacertada la dependencia total de los hidrocarburos, representando el petróleo un 95,8\% de sus exportaciones lo que se explicó en párrafos anteriores, cuando se analizó de que la balanza Comercial de 
este país presenta cifras aceptadas dentro del rango normal (Ver gráfico 5), debido precisamente a que este acervo de petrodólares eran mucho mayores que las importaciones de esa época; pero desgraciadamente se concluye que no aprovecharon esta bonanza, puesto que no se guardaron reservas para enfrentar las contingencias que hoy presentan la significativa baja en los precios de este oro negro, lo cual impidió los ajuste macroeconómicos que hoy día está demandando la precaria economía de este país. Esta situación sucedida en Venezuela en cuanto al no aprovechamiento de los petrodólares, se explica con un pasaje de la biblia, en el cual José le interpretó un sueño al faraón, y le dijo que se debían aprovechar las vacas gordas, para la época de las vacas flacas, medida sabia de José, hijo de Jacob, y que permitió que Egipto fuera la potencia de esa época, porque al presentarse la época de escasez en todos los reinos, este país tenía las reservas más grandes de toda la región, y tan efectiva fue esta sugerencia de José, que le dio méritos para que el Faraón lo nombrara Gobernador de este país, (Génesis 41, 1:56)

Ya en 2016 como consecuencia de todo lo anterior, Venezuela presenta un déficit fiscal estimado por encima de 19,89\% de su PIB, al igual que una considerable necesidad de financiación externa; y además, no se ha podido apaciguar la fuga de divisas.

El reto que debe enfrentar Venezuela consiste en contener los desbalances de las decisiones de política macroeconómicas que han echado por el suelo los logros obtenidos en décadas pasadas, y recuperar la confianza del sector privado, optimizando el clima para las inversiones, orientándolas a fortalecer sus deseos de desarrollo a largo plazo, y diversificar sus exportaciones, a fin de reducir su dependencia de los riesgos que ocasiona las constantes bajas de los precios del petróleo; todo estos se debe acompañar con la implementación de estrategias tendientes a proteger a la población inmersa en un ambiente de pobreza.

Todo esto como se comentó anteriormente, unido a las medidas cambiarias y la reducción casi total de la contribución del sector privado en la producción y distribución de algunos productos básicos, han originado una escasez de bienes y servicios, amenazas inflacionarias y problemas de abastecimiento en un aparato productivo altamente dependiente de las importaciones. La inflación ha continuado acelerándose a lo largo 
del año, llegando a 790\% en 2017(Ver gráfico4), mientras que el Bolívar ha continuado depreciándose significativamente en el mercado negro, tal es el caso que sucede en Colombia, en donde se observa que muchos venezolanos inmigrantes, se montan en los transportes urbanos y mendigan una limosna a cambio de fajos de billetes de 100 bolívares.

Todas estas circunstancias unidas a políticas distorsionadas, encaminada a restricciones en el margen de beneficios y el desestimulo a la inversión, como por ejemplo, han ocasionado un ambiente de incertidumbre y desconfianza, lo cual deprimió el consumo interno, la inversión privada, la disponibilidad de bienes de capital, y como consecuencia la inevitable contracción extrema de esta economía, que en otrora fue una de las mejores de Latinoamérica y del mundo.

\section{Referencias Bibliográficas}

Banco Mundial (2016). PIB de Bolivia. Recuperado el 21 de Enero de 2017, disponible en: https://datos.bancomundial.org/pais/bolivia.

Banco Central de Perú (2017). Estadísticas. Recuperado Febrero 1 de 2018, disponible en: http://www.bcrp.gob.pe/estadisticas.html

Banco Central de Ecuador. Indicadores Económicos. Recuperado el 2 de Febrero, disponible en: https://www.bce.fin.ec/index.php/indicadores-economicos

Banco Mundial (2017). El Banco Mundial en Venezuela. Recuperado el día 3 de Febrero de 2018, disponible en: http://www.bancomundial.org/es/country/ venezuela

Banco Mundial (2017). ¿Qué es producto interno bruto PIB?. Recuperado el día 4 de Febrero de 2018, disponible en: http://www.banrep.gov.co/es/ contenidos/page/qu-producto-interno-bruto-pib

Banco Mundial (2018). El Banco Mundial en Perú. Recuperado el día 9 de Mayo de 2018, en: http://www.bancomundial.org/es/country/peru

Caballero, A. (2017). Análisis del Crecimiento de las Economía Emergentes y Desarrolladas Antes y Después de la Primera Crisis Mundial del Siglo XXI. Revista Universitaria Ruta.19(6), 90-116.

Castro, A. (2014). La convivencia y la mediación de conflictos como estrategia pedagógica en la vida escolar. Panorama Económico 22 (1), 169-190 
CELAG (2016). Salvaguardias revirtieron el déficit de la balanza comercial en Ecuador. Recuperado el 7 de Febrero, disponible en: http://www.eltelegrafo. com.ec/noticias/economia/8/salvaguardias-y-acuerdo-multipartesrevirtieron-el-deficit-de-la-balanza-comercial

CEPAL (2016). Plan de Desarrollo Económico y Social en el marco del Desarrollo Integral para Vivir Bien (PDES) 2016-2020 de Bolivia. Recuperado el 10 de Febrero de 2018, disponible en: https://observatorioplanificacion.cepal. org/es/planes/plan-de-desarrollo-economico-y-social-en-el-marco-deldesarrollo-integral-para-vivir-bien

CEPAL (2015).Plan Estratégico de Gobierno 2015-2019 “Un sólo país" de Panamá. Recuperado el 11 de Febrero de 2018, disponible en: https:// observatorioplanificacion.cepal.org/es/planes/plan-estrategico-degobierno-2015-2019-un-solo-pais-de-panama

DANE (2017). Indicadores Económicos. Recuperado el 8 de febrero de 2018, disponible en: http://www.dane.gov.co/index.php/67-espanol/serviciosdane/indicadores- económicos.

Datos macro. Producto interno Bruto. Recuperado el 3 de Febrero de 2018, disponible en: https://www.datosmacro.com/pib/colombia

De Ycaza, Á. (2016). El manejo de la balanza de pagos en Panamá. Recuperado el 12 de Febrero de 2018, disponible en: http://laestrella.com.pa/economia/ manejo-balanza-pagos-panama/23976277

Dinero (2017). ¿Qué pasará con la economía ecuatoriana tras el terremoto?. Recuperado el día 6 de Mayo de 2018, en: https://www.dinero.com/edicionimpresa/mundo/articulo/las-implicaciones-sociales-y-economicas-trasterremoto-de-ecuador/222948

El Economista (2014). Así es la historia del desplome del petróleo: una caída del $50 \%$ en 6 meses tiene explicación. Recuperado el dia 6 de febrero, y disponible en: http://www.eleconomistaamerica.co/noticias/ noticias/6333888/12/14/Asi-es-la-historia-del-desplome-del-petroleouna-caida-del-50-en-6-meses-tiene-explicacion.html

Forbes (2017).Reducción de la pobreza en Panamá es mínima. Recuperado el día 11 de febrero de 2018, disponible en: https://www.forbes.com.mx/ reduccion-la-pobreza-panama-minima/

Giuman, L. (2017). Economía venezolana, un modelo fuera de control. Recuperado el 19 de Febrero de 2018, disponible en: http://www.semana.com/mundo/ articulo/crisis-economica-en-venezuela/532871 
Gómez, M. (2017). Así está Colombia, un año después de las Farc. Recuperado el 7 de mayo de 2018, en : http://www.eltiempo.com/politica/proceso-de-paz/ efectos-en-colombia-tras-un-ano-de-la-firma-del-acuerdo-de-paz-con-lasfarc- 152740

Hernández, J. (2015). Desafíos y responsabilidades de la profesión contable frente a la contabilidad ambiental. Revista Aglala. 6(7), 164-182.

Herrera, V., B. \& Caballero B., J. (2002). "Dolarizar: ¿realmente un dilema?". Cuadernos de Economía No. 36, Bogotá.

INEI (2017). Pobreza extrema en el Perú cayó 0.3\% en 2016, Recuperado el 9 de Febrero de 2018, disponible en: https://peru21.pe/economia/pobrezaextrema-peru-cayo-0-3-2016-inei-75910

Índex Mundi. Listado de países. Recuperado el día 4 de Febrero de 2018, disponible en: https://www.google.com.co/search?ei=VDBzWsW6JMie5gLU47BY\&q=I ndex+Mundi\&oq=Index+Mundi\&gs_l=psy-ab.3..0i10k112j0i10i67k1j0i10k1l 7.2480.7852.0.14527.53.21.0.0.0.0.273.2648.0j15j1.17.0...0...1.1.64.psy-ab.. 41.11.1818.0..0j33i21k1j0i131k1j0i67k1j0i131i67k1.136.hMUiyquF0Qk

La Biblia (2017). Dios habla Hoy. Recuperado el 12 de Febrero de 2018, disponible en: https://www.bible.com/es/bible/1846/GEN.24.DHHS94

Muñoz de Bustillo, R. (2002). "Reflexiones sobre la Dolarización". Universidad de Salamanca. Claves de Economía Mundial, pp. 422-429; ICEX. Madrid.

Olmos, G. (2017). La nueva guerra del gas: ¿por qué las políticas de Donald Trump son una amenaza para la economía de Bolivia?. Recuperado el dia 30 de Abril de 2018 en: http://www.bbc.com/mundo/noticias-internacional-42074586

Programa de las Naciones Unidas para el Desarrollo PNUD (2016).Informe sobre Desarrollo Humano. Recuperado el 6 de Febrero de 2018, disponible en: http://hdr.undp.org/sites/default/files/HDR2016_SP_Overview_Web.pdf.

Perdomo, M. (2016). Las cifras del TLC con Estados Unidos confirman su fracaso. Recuperado el 4 de Febrero de 2018, disponible en:https://jorgerobledo. com/las-cifras-del-tlc-con-estados-unidos-confirman-su-fracaso/

Perfetti, M. (2014). Así se calculan las cifras de desempleo en Colombia. Recuperado el 7 de Febrero de 2018, disponible en: http://www.dinero. com/pais/articulo/como-mide-dane-cifras-desempleo/201279

Quispe, M. (2017). Impacto de los programas sociales en la disminución de la pobreza. Revista Pensamiento Crítico. 22(5), 69-102

Rojas, B. (2014). Desempleo en Bolivia se reduce pero crece trabajo informal. Recuperado el día 7 de Febrero de 2018, disponible en: https://www. 
americaeconomia.com/economia-mercados/finanzas/desempleo-enbolivia-se-reduce-pero-crece-trabajo-informal

Sarmiento, S. (2017). De Hoover a Trump. Recuperado el 7 de febrero de 2018, disponible en: http://diario.mx/Opinion/2017-01-24_162dc8ed/de-hoovera-trump---/

Schuler, K. (1999). "Fundamentos de la Dolarización". Reporte del Comité de Asuntos Económicos del Congreso de los Estados Unidos. Recuperado el 5 Mayo de 2018, en: http://users.erols.com/kurrency/basicssp.htm

Varela, J. (2016). Panamá pide a Colombia aceptar "de una vez por todas" fallo de OMC, Recuperado el dia 1 de Mayo de 2018 en: https://www.semana. com/economia/articulo/panama-pide-a-colombia-aceptar-de-una-vez-portodas-fallo-de-omc/484322 